International Journal of Pure and Applied Mathematics

Volume 88 No. 4 2013, 529-536

ISSN: 1311-8080 (printed version); ISSN: 1314-3395 (on-line version)

url: http://www.ijpam.eu

doi: http://dx.doi.org/10.12732/ijpam.v88i4.7

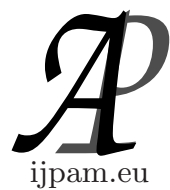

\title{
UNIQUENESS OF FRACTIONAL DIFFERENTIAL EQUATION USING THE INEQUALITIES
}

\author{
Vaijanath L. Chinchane ${ }^{1 \S}$, Deepak B. Pachpatte ${ }^{b}$ \\ ${ }^{1}$ Department of Mathematics \\ Deogiri Institute of Engineering and Management \\ Studies Aurangabad, 431005, INDIA \\ ${ }^{2}$ Department of Mathematics \\ Dr. Babasaheb Ambedkar Marathwada \\ University, Aurangabad, 431 004, INDIA
}

\begin{abstract}
In the present paper, we study the uniqueness theorem of solution of fractional differential equation with initial condition by using Bihari's and Medved inequality.
\end{abstract}

AMS Subject Classification: 26D10, 26A33, 34A08, 34A12

Key Words: fractional derivative, fractional integral, Bihari's inequality and Medved inequality

\section{Introduction}

Recently, fractional differential equations have been used in physical science and engineering application $[2,3,10]$. Fractional differential equation is generalization of ordinary differential equation of fractional order (i.e non-integer order). Fractional differential equation have many applications in various fields of science and engineering such as electrochemistry, viscoelasticity, electromagnetic, control theory, porous media $[2,6,10]$. In the last few decades, many researcher have studied the existence and uniqueness of the initial value problem for fractional differential equation, see $[2,3,5,7,10,13]$. In this paper we

Received: August 27, 2013

(c) 2013 Academic Publications, Ltd.

$\S_{\text {Correspondence author }}$ url: www.acadpubl.eu 
consider the initial valve problem of the type

$$
\begin{gathered}
D^{\alpha} u(t)=f(t, u(t)), \\
\left.D^{\alpha-1} u(t)\right|_{t=0}=\eta,
\end{gathered}
$$

where $0<\alpha<1, t \in[0, \infty)$, and $f$ is real valued function on $D=I \times R$ into $R$; where $\mathrm{R}$ denote the real space and $\eta$ is real constant, $D^{\alpha}$ denote the Riemann-Liouville derivative operator.

\section{Preliminaries}

The necessary details of fractional calculus are given in the book I. Podlubny [10], A.A.Kilbas [3], and in book of S.G.Samko et al.[11], here we present some notation and definitions of Riemann-Liouville derivative and integral as given in $[10,12]$.

Definition 2.1. The fractional derivative of order $0<\alpha<1$ of continuous function $f: R^{+} \longrightarrow R$, by

$$
D^{\alpha} f(x)=\frac{1}{\Gamma(1-\alpha)} \frac{d}{d x} \int_{0}^{x}(x-t)^{-\alpha} f(t) d t,
$$

provided that the right side is pointwise defined on $R^{+}$.

Definition 2.2. The fractional primitive of order $\alpha>0$, of function $f: R^{+} \longrightarrow R$ is given as follows

$$
I^{\alpha} f(x)=\frac{1}{\Gamma(\alpha)} \int_{0}^{x}(x-t)^{\alpha-1} f(t) d t .
$$

provided that the right side is pointwise defined on $R^{+}$, where $\Gamma(\alpha)=$ $\int_{0}^{\infty} e^{-u} u^{\alpha-1} d u$.

In [10], the author have been studied the existence and uniqueness of the initial value problem (1.1)-(1.2), first, let us reduce the problem (1.1)-(1.2)to a fractional integral equation, we obtain

$$
u(t)=\frac{\eta}{\Gamma(\alpha)} t^{\alpha-1}+\frac{1}{\Gamma(\alpha)} \int_{0}^{t}(t-\tau)^{\alpha-1} f(t, u(\tau)) d \tau .
$$

It is clear that equation (2.3) is equivalent to the initial problem (1.1)-(1.2), see [10,pp129-128]. 
Also, in [9] Medved defined a special class of nonlinear function and developed a method to estimate solution for nonlinear integral inequalities with singular kernel. The class of function defined as follows:

Definition 2.3. [9] Let $q>0$ be a real number and $0<T \leq \infty$, we say that a function $w: R_{+} \longrightarrow R$ satisfies a condition (q) if

$$
e^{-q t}[w(u)]^{q} \leq R(t) w\left(e^{-q t} u^{q}\right)
$$

for all $u \in R_{+}, t \in[0, T)$, where $R(t)$ is continues nonnegative function.

Remark 2.1. [9] If $w(u)=u^{m}, m>o$ then

$$
e^{-q t}[w(u)]^{q}=e^{(m-1) q t} w\left(e^{-q t} u^{q}\right)
$$

for any $q>1$. i.e the condition $(q)$ is satisfies with $R(t)=e^{(m-1) q t}$. for $w(u)=u+a u^{m}$, where $0 \leq a \leq 1, m \geq 1$ the function w satisfies the condition (q) with $q>1$ and $R(t)=2^{q-1} e^{q m t}$.

Theorem 2.1. [9] Let $0 \geq T \leq \infty, u(t), b(t), a(t), a^{\prime}(t) \in C\left([0, T), R_{+}\right)$; $w \in C\left(R_{+}, R\right)$ be nondecreasing function, $w(0)=0, w(u)>0$ on $(0, T)$, and

$$
u(t) \leq a(t)+\int_{0}^{t}(t-s)^{\beta-1} b(s) w(u(s)) d s,
$$

for $t \in[0, T)$ where $\beta>0$ is constant. Then following hold:

(i) Suppose $\beta>\frac{1}{2}$, and $w$ satisfies the condition (q) with $q=2$, then

$$
u(t) \leq e^{t}\left\{\Omega^{-1}\left[\Omega\left(2 a(t)^{2}\right)+g_{1}(t)\right]\right\}^{\frac{1}{2}},
$$

for $t \in[0, T]$, where

$$
g_{1}(t)=\frac{\Gamma(2 \beta-1)}{4^{\beta-1}} \int_{0}^{t} R(t) b(s)^{2} d s,
$$

where $\Gamma$ is gamma function, $\Omega(v)=\int_{v_{0}}^{v} \frac{d s}{w(s)}, v_{0}>0, \Omega^{-1}$, is the inverse of $\Omega$, and $t \in R_{+}$is such that $\Omega\left(2 a(t)^{2}\right)+g_{1}(t) \in \operatorname{Dom}\left(\Omega^{-1}\right)$ for all $t \in[0, T]$. (ii)Let $\beta=\left(0, \frac{1}{2}\right]$ and $w$ satisfies the condition $(q)$ with $q=z=2$, where $z=\frac{1-\beta}{\beta}$, i.e $\beta=\frac{1}{z+1}$. Let $\Omega, \Omega^{-1}$ be as in part (i). Then

$$
u(t) \leq e^{t}\left\{\Omega^{-1}\left[\Omega\left(2^{q-1} a(t)^{2}\right)+g_{2}(t)\right]\right\}^{\frac{1}{q}},
$$

for $t \in[0, T]$, where

$$
g_{2}(t)=2^{q-1} K_{z}^{q} \int_{0}^{t} R(s) b(s)^{2} d s
$$




$$
K_{z}=\left[\frac{\Gamma(1-\alpha p)}{p^{1-\alpha p}}\right]^{\frac{1}{p}}, \quad \alpha=\frac{z}{z+1}, \quad p=\frac{z+2}{z+1},
$$

and $T_{1} \in R_{+}$is such that $\Omega\left(2^{q-1} a(t)^{q}\right)+g_{2}(t)$ for all $t \in[0, T]$.

Theorem 2.2. [9] Let $\geq T \leq \infty, u(t), b(t), a(t), a^{\prime}(t)$ be as in theorem (2.1) and

$$
u(t) \leq a(t)+\int_{0}^{t}(t-s)^{\beta-1} b(s) u(s) d s,
$$

for $t \in[0, T)$ where $\beta>0$ is constant. Then following hold:

(i) Suppose $\beta>\frac{1}{2}$, then

$$
u(t) \leq(\sqrt{2}) a(t) \exp \left[\frac{2 \Gamma(2 \beta-1)}{4^{\beta}} \int_{0}^{t} b(s)^{2} d s+t\right],
$$

for $t \in[0, T]$, (ii)If $\beta=\frac{1}{z+1}$, for some $z \geq 1$. Then

$$
u(t)=\left(2^{q-1}\right)^{\frac{1}{q}} a(t) \exp \left[\frac{2^{q-1}}{q} K_{z}^{q} \int_{0}^{t} b(s)^{2} d s+t\right],
$$

for $t \in[0, T]$, where $K_{z}$ is defined by (2.7), $q=z+2$.

For detail proof of above two theorem see [9].

Theorem 2.3. (Bihari's inequality) $[1,8]$ Let $u$ and $f$ be nonnegative defined on $R_{+}$, let $w(u)$ be continuous nondecreasing function defined on $R_{+}$, and $w(u)>0$, on $(0, \infty)$. If

$$
u(t) \leq k+\int_{0}^{t} f(s) w(u(s)) d s,
$$

for $t \in R_{+}$where $k$ is nonnegative constant, for $0 \leq t \leq T$

$$
u(t) \leq G^{-1}\left[G(k)+\int_{0}^{t} f(s) d s\right]
$$

where,

$$
G(r)=\int_{0}^{t} \frac{d s}{w(s)}, r>0, r_{0}>0
$$

and $G^{-1}$ is the inverse function of $G$ and $t_{1} \in R_{+}$is chosen so that

$$
G(k)+\int_{0}^{t} f(s) d s \in \operatorname{Dom}\left(G^{-1}\right) .
$$

For all $t \in R_{+}$laying in the interval $0 \leq t \leq t_{1}$. 


\section{Main Result}

In this section, we establish the uniqueness of solution of the following initial value problem.

Theorem 3.1. Consider the fractional differential equation

$$
D^{\alpha} u(t)=f(t, u(t))
$$

with initial condition

$$
\left.D^{\alpha-1} u(t)\right|_{t=0}=\eta
$$

where $f(t, u(t)):[0, T) \times R \longrightarrow R$ is continuous on $I=[0, T)$ and satisfies Lipschitz condition with respect to second variable i.e

$$
|f(t, u(t))-f(t, v(t))| \leq M \Phi(|u(t)-v(t)|) .
$$

If $\Phi(u)$ is a continuous nondecreasing function on $0<u \leq A$, with $\Phi(0)=0$ and

$$
\int_{0}^{A} \frac{d u}{\Phi(u)}
$$

then the solution of problem (3.1)-(3.2) is unique.

Proof. Assume that there exists two solution $u(t)$ and $v(t)$ of (3.1)-(3.2), we have

$$
\begin{aligned}
& u(t)=\frac{\eta}{\Gamma(\alpha)} t^{\alpha-1}+\frac{1}{\Gamma(\alpha)} \int_{0}^{T}(t-\tau)^{\alpha-1} f(t, u(\tau)) d \tau \\
& v(t)=\frac{\eta}{\Gamma(\alpha)} t^{\alpha-1}+\frac{1}{\Gamma(\alpha)} \int_{0}^{T}(t-\tau)^{\alpha-1} f(t, v(\tau)) d \tau
\end{aligned}
$$

Which lead easily to

$$
\begin{aligned}
&|u(t)-v(t)| \leq \frac{1}{\Gamma(\alpha)} \int_{0}^{T}(t-\tau)^{\alpha-1}|f(t, u(\tau))-f(t, v(\tau))| d \tau \\
& \leq \frac{1}{\Gamma(\alpha)} \int_{0}^{T}(t-\tau)^{\alpha-1} \Phi(|u(\tau)-v(\tau)|) d \tau \\
&|u(t)-v(t)|<\epsilon+\frac{L}{\Gamma(\alpha)} \int_{0}^{T}(t-\tau)^{\alpha-1} \Phi(|u(\tau)-v(\tau)|) d \tau
\end{aligned}
$$


Now, we can apply Bihari's inequality to (3.7) getting,

$$
\begin{aligned}
|u(t)-v(t)|< & \Phi^{-1}\left[\Phi(\epsilon)+\frac{M L}{\Gamma(\alpha)} \int_{0}^{T}(t-\tau)^{\alpha-1} d \tau\right] \\
& <\Phi^{-1}\left[\Phi(\epsilon)+\frac{M L}{\Gamma(\alpha)}\left(\frac{(t-\tau)^{\alpha}}{-\alpha}\right)_{0}^{T}\right] \\
& <\Phi^{-1}\left[\Phi(\epsilon)+\frac{M L}{\Gamma(\alpha)}\left(\frac{T^{\alpha}}{\alpha}-\frac{(t-T)^{\alpha}}{\alpha}\right)\right] \\
& <\Phi^{-1}\left[\Phi(\epsilon)+\frac{M L}{\Gamma(\alpha+1)}\left(T^{\alpha}-(t-T)^{\alpha}\right)\right]
\end{aligned}
$$

where $\Phi(u)$ is primitive for $\frac{1}{\Phi(u)}$. We shall prove that the right-hand side of (3.8)tends toward zero. as $\varepsilon \rightarrow 0$. As $|u(t)-v(t)|$ is independent of $\varepsilon$, it follows that $u(t)=v(t)$, which we need. Let us remark that condition (3.4) implies $\Phi(\varepsilon) \rightarrow-\infty$ for $\varepsilon \rightarrow 0$, no matter how we choose the primitive of $\frac{1}{\Phi(u)}$. Thus $\Phi^{-1}(u) \rightarrow o$ as $u \rightarrow-\infty$. Consequently, $\varepsilon \rightarrow 0$ in the inequality (3.8), the right-hand side tends toward zero. Thus theorem is proved.

Theorem 3.2. The initial value problem (3.1)-(3.2) has unique solution in the interval $t \in[0, T)$. If the function $f$ is continuous and satisfies the Lipschitz condition with respect to second variable i.e

$$
|f(t, u(t))-f(t, v(t))| \leq M|u(t)-v(t)|,
$$

for some positive constant $M$.

Proof. Assume that $u(t)$ and $v(t)$ be two solution of initial value problem (3.1)-(3.2), we have

$$
\begin{aligned}
& u(t)=\frac{\eta}{\Gamma(\alpha)} t^{\alpha-1}+\frac{1}{\Gamma(\alpha)} \int_{0}^{T}(t-\tau)^{\alpha-1} f(t, u(\tau)) d \tau \\
& v(t)=\frac{\eta}{\Gamma(\alpha)} t^{\alpha-1}+\frac{1}{\Gamma(\alpha)} \int_{0}^{T}(t-\tau)^{\alpha-1} f(t, v(\tau)) d \tau
\end{aligned}
$$

which gives,

$$
\begin{aligned}
|u(t)-v(t)| \leq & \frac{1}{\Gamma(\alpha)} \int_{0}^{T}(t-\tau)^{\alpha-1}|f(t, u(\tau))-f(t, v(\tau))| d \tau \\
& \leq \frac{M}{\Gamma(\alpha)} \int_{0}^{T}(t-\tau)^{\alpha-1} 1|u(\tau)-v(\tau)| d \tau \\
& \leq \varepsilon+\frac{M}{\Gamma(\alpha)} \int_{0}^{T}(t-\tau)^{\alpha-1} 1|u(\tau)-v(\tau)| d \tau
\end{aligned}
$$


Now, $(i)$ suppose that $\alpha>\frac{1}{2}$, then applying theorem (2.2) (i) to (3.12), we have

$$
|u(t)-v(t)|<\frac{\sqrt{2 \varepsilon M}}{\Gamma(\alpha)} \exp \left[\frac{2 \sqrt{2 \alpha-1}}{4^{\alpha}} t+t\right],
$$

for $t \in[0, T)$ since $\varepsilon$ is arbitrary, as $\varepsilon \rightarrow 0$ the inequality (3.13) implies that $u(t)=v(t)$ on $[0, T)$.

(ii) Let $\alpha>\frac{1}{z+1}$ for some $z \geq 1$, then apply the theorem (2.2)(ii) to (3.13), again we have,

$$
|u(t)-v(t)|<\frac{\left(2^{q-1}\right)^{\frac{1}{q}} \varepsilon M}{\Gamma(\alpha)} \exp \left[\frac{2^{q-1}}{q} K_{z}^{q} t+t\right],
$$

for $t \in[0, T)$ where $K_{z}$ is defined by (2.7). Since $\varepsilon$ is arbitrary in (3.14) implies that $u(t)=v(t)$. Hence the theorem is proved.

\section{References}

[1] C. Corduneanu, Principal of Differential and Integral Equation, Allynand Bacon, Boston, 1971.

[2] R. Hilfer, Application of Fractional Calculus in Physics, World Scientific, Singapore, 2000.

[3] A. A. Kilbas, H. M. Srivastava and J. J. Trujillo, Theory and Application of Fractional Differential Equations, Elsevier, Amersterdam, 2006.

[4] V. Lakshmikantam and A.S. Vatsala, Basic theory of fractional Differential Equations, Nonlinear Analysis, 69 No. 8 (2008), 2677-2682., doi:10.1016/j.na.2007.08.042.

[5] V. Lakshmikantam, Theory of Fractional functional Differential Equations, Nonlinear Analysis, 69 No. 10 (2008), 3337-3343., doi:10.1016/j.na.2007.09.025.

[6] F. Mainardi, Fractional Calculus: some basic problem in continuum and stastical mechanics, in factals and fractional calculus in contiuam mechanics, (A.Carpinteri and F.Mainardi, Eds)Springer-Verlag, Wien, (1997) 291348.

[7] S. M. Momani, Local and global uniqueness theorem on differential equations of non-integer order via Bihari's and Gronwall inequalities, Rev. Tec. Ing. Univ. Zulia. Vol. 23, No.1, (2000) 66-69. 
[8] B. G. Pachpatte, Inequalities for Differential and Integral Equations, Academic Press, New York, 205, 1998.

[9] B. G. Pachpatte, Integral and finite difference inequalities and applications, North Holland Mathematics Studies, 205, Elsevier Science, 2006.

[10] I. Podlubny, Fractional Differential Equations, Academic Press, New York, 1999.

[11] S. G. Somko, A. A. Kilbas and O. I. Marichev, Fractional Integral and Derivative Theory and Application, Gordon and Breach, Yverdon, Switzerland, 1993.

[12] Haping Ye, Jianming Gao and Yongs Ding, A generalized Grownwall inequality and it's application to fractional differential equation, Journal of Mathematical Analysis and Application. 328 No 2 (2007), 1075-1081., doi:10.1016/j. jmaa.2006.05.061.

[13] Yong. Zhou, Existence and uniqueness of solution for system of fractional differential equations, Fractional Calculus and Applied Analysis. 12, No.2, 2009 . 